Article

\title{
Casorati Inequalities for Submanifolds in a Riemannian Manifold of Quasi-Constant Curvature with a Semi-Symmetric Metric Connection
}

\author{
Pan Zhang ${ }^{1, *}$ and Liang Zhang ${ }^{2}$ \\ 1 School of Mathematical Sciences, University of Science and Technology of China, Anhui 230026, China \\ 2 School of Mathematics and Computer Science, Anhui Normal University, Anhui 241000, China; \\ zhliang43@ahnu.edu.cn \\ * Correspondence: panzhang@mail.ustc.edu.cn; Tel.: +1-865-595-1891
}

Academic Editor: Kenneth Berenhaut

Received: 10 December 2015; Accepted: 25 March 2016; Published: 31 March 2016

\begin{abstract}
By using new algebraic techniques, two Casorati inequalities are established for submanifolds in a Riemannian manifold of quasi-constant curvature with a semi-symmetric metric connection, which generalize inequalities obtained by Lee et al. J. Inequal. Appl. 2014, 2014, 327.
\end{abstract}

Keywords: Casorati inequalities; submanifolds; quasi-constant curvature; semi-symmetric metric connection

MSC: 53C40; 53B05; 53B15

\section{Introduction}

In 1996, Chen initiated the following fundamental problem in his cornerstone work [1]: to establish simple relationships between the main intrinsic invariants and the main extrinsic invariants of Riemannian submanifolds. The basic relationships discovered until now are inequalities and the study of this topic has attracted a lot of attention during the last two decades. We refer to [2] for a relatively recent survey on this topic.

On the other hand, Casorati introduced the Casorati curvature of an $n$-dimensional submanifold $M$ of a Riemannian manifold, which is an extrinsic invariant defined as the normalized square of the length of the second fundamental form of the submanifold [3]. In 2007, Decu et al. introduced the normalized $\delta$-Casorati curvatures $\delta_{c}(n-1)$ and $\hat{\delta}_{c}(n-1)$ and established inequalities involving $\delta_{\mathcal{c}}(n-1)$ and $\hat{\delta}_{\mathcal{c}}(n-1)$ for submanifolds in real space forms [4].

The proof of the inequalities in [4] is based on an optimization procedure by showing that the quadratic polynomial in the components of the second fundamental form is parabolic. And the above method was successfully applied to establish inequalities in terms of the Casorati curvatures for different submanifolds in various ambient spaces [5-10]. Recently, in [11-13], the authors obtained the corresponding Casorati inequalites by using Oprea's optimization methods on Riemannian submanifolds [14].

In [15,16], Mihai and Özgür established Chen inequalities for submanifolds of real, complex and Sasakian space forms endowed with semi-symmetric metric connections and in [17,18], Özgür and Murathan gave Chen inequalities for submanifolds of a locally conformal almost cosymplectic manifold and a cosymplectic space form endowed with semi-symmetric metric connections. On the other hand, Lee et al. proved inequalities involving the Casorati curvature of submanifolds in real, complex and Sasakian space forms endowed with a semi-symmetric metric connection in [7,8]. In an earlier paper, Özgür established Chen inequalities for submanifolds in a Riemannian manifold of 
quasi-constant curvature [19]. Just very recently, we obtained Chen's inequalities for submanifolds of a Riemannian manifold of quasi-constatnt curvature with a semi-symmetric metric connection [20].

In this paper, by using new algebraic techniques, we establish inequalities involving the normalized $\delta$-Casorati curvatures $\delta_{c}(n-1)$ and $\hat{\delta}_{c}(n-1)$ for submanifolds in a Riemannian manifold of quasi-constant curvature with a semi-symmetric metric connection, which generalize inequalities obtained in [7]. Our techniques can also be used to establish inequalities involving the generalized normalized $\delta$-Casorati curvatures obtained in $[5,9]$.

\section{Preliminaries}

To meet the requirements in the next sections, here, we briefly present the basic elements of the theory of a Riemannian manifold endowed with a semi-symmetric metric connection.

Let $N^{n+p}$ be an $(n+p)$-dimensional Riemannian manifold with the Riemannian connection $\hat{\nabla}$, a linear connection $\bar{\nabla}$ and the Riemannian metric $g$. The torsion tensor field $\bar{T}$ of the linear connection $\bar{\nabla}$ is defined by

$$
\bar{T}(\bar{X}, \bar{Y})=\bar{\nabla}_{\bar{X}} \bar{Y}-\bar{\nabla}_{\bar{Y}} \bar{X}-[\bar{X}, \bar{Y}]
$$

for the vector fields $\bar{X}, \bar{Y}$ on $N^{n+p}$.

The liner connection $\bar{\nabla}$ is said to be semi-symmetric if the torsion tensor $\bar{T}$ of the connection $\bar{\nabla}$ satisfies the following relation

$$
\bar{T}(\bar{X}, \bar{Y})=\phi(\bar{Y}) \bar{X}-\phi(\bar{X}) \bar{Y}
$$

for a 1 -form $\phi$ on $N^{n+p}$. Further, if $\bar{\nabla}$ satisfies $\bar{\nabla} g=0$, then $\bar{\nabla}$ is called a semi-symmetric metric connection [21]. In [21], Yano obtained a relation between the semi-symmetric metric connection $\bar{\nabla}$ and the Riemannian connection $\hat{\nabla}$ which is given by

$$
\bar{\nabla}_{\bar{X}} \bar{Y}=\hat{\nabla}_{\bar{X}} \bar{Y}+\phi(\bar{Y}) \bar{X}-g(\bar{X}, \bar{Y}) P
$$

where $P$ is a vector field defined by

$$
g(P, \bar{X})=\phi(\bar{X})
$$

for any vector field $\bar{X}$ on $N^{n+p}$.

Let $M^{n}$ be an $n$-dimensional submanifold of an $(n+p)$-dimensional Riemannian manifold $N^{n+p}$ with the semi-symmetric metric connection $\bar{\nabla}$ and the Riemannian connection $\hat{\bar{\nabla}}$. On $M^{n}$ we consider the induced semi-symmetric metric connection denoted by $\nabla$ and the induced Riemannian connection denoted by $\hat{\nabla}$.

Let $\bar{R}$ be the curvature tensor of $N^{n+p}$ with respect to $\bar{\nabla}$ and $\hat{R}$ the curvature tensor of $N^{n+p}$ with respect to $\hat{\nabla}$. We also denote by $R$ and $\hat{R}$ the curvature tensors associated to $\nabla$ and $\hat{\nabla}$, respectively, on $M^{n}$.

The Gauss formulas with respect to $\nabla$, respectively $\hat{\nabla}$, can be written as the following [22]

$$
\bar{\nabla}_{X} Y=\nabla_{X} Y+h(X, Y), \hat{\nabla}_{X} Y=\hat{\nabla}_{X} Y+\hat{h}(X, Y)
$$

for any vector fields $X, Y$ on $M$, where $h$ is a $(0,2)$ symmetric tensor on $M^{n}$ and $\hat{h}$ is the second fundamental form associated to Riemaniann connection $\hat{\nabla}$. According to the formula (7) from [22] $h$ is also symmetric.

The curvature tensor $\bar{R}$ with respect to the semi-symmetric metric connection $\bar{\nabla}$ on $N^{n+p}$ can be written as [23]

$$
\begin{aligned}
\bar{R}(X, Y, Z, W)= & \hat{R}(X, Y, Z, W)+\alpha(Y, Z) g(X, W)-\alpha(X, Z) g(Y, W) \\
& +\alpha(X, W) g(Y, Z)-\alpha(Y, W) g(X, Z)
\end{aligned}
$$


for any vector fields $X, Y, Z, W$ on $M^{n}$, where $\alpha$ is a $(0,2)$-tensor field defined by

$$
\alpha(X, Y)=\left(\hat{\nabla}_{X} \phi\right) Y-\phi(X) \phi(Y)+\frac{1}{2} \phi(P) g(X, Y)
$$

denote by $\lambda$ the trace of $\alpha$ restricted on $M^{n}$.

The Gauss equation for the submanifold $M^{n}$ into $N^{n+p}$ with respect to the semi-symmetric metric connection is given by Nakao [22]

$$
R(X, Y, Z, W)=\bar{R}(X, Y, Z, W)+g(h(X, Z), h(Y, W))-g(h(X, W), h(Y, Z))
$$

for any vector fields $X, Y, Z, W$ on $M^{n}$.

According to the Equation (7) from [22] we have

Lemma 1. If $P$ given by Equation (1) is a tangent vector field on $M^{n}$, then $h=\hat{h}$.

In $N^{n+p}$ we choose a local orthonormal frame $e_{1}, \cdots, e_{n}, e_{n+1}, \cdots, e_{n+p}$, such that, restricting to $M^{n}, e_{1}, \cdots, e_{n}$ are tangent to $M^{n}$.

Let $\pi \subset T_{x} M^{n}, x \in M^{n}$, be a 2-plane section. Denote by $K(\pi)$ the sectional curvature of $M^{n}$ with respect to the induced semi-symmetric metric connection. Then the scalar curvature $\tau$ with respect to the semi-symmetric metric connection is defined by

$$
\tau(x)=\sum_{1 \leq i<j \leq n} K\left(e_{i} \wedge e_{j}\right)
$$

and the normalized scalar curvature $\rho$ with respect to the semi-symmetric metric connection is defined by

$$
\rho=\frac{2 \tau}{n(n-1)}
$$

We write

$$
\hat{h}_{i j}^{r}=g\left(\hat{h}\left(e_{i}, e_{j}\right), e_{r}\right), \quad h_{i j}^{r}=g\left(h\left(e_{i}, e_{j}\right), e_{r}\right)
$$

Then we denote the Casorati curvature with respect to the semi-symmetric metric connection by

$$
\mathcal{C}=\frac{1}{n} \sum_{r=n+1}^{n+p} \sum_{i, j=1}^{n}\left(h_{i j}^{r}\right)^{2}
$$

Suppose now that $L$ is an $l$-dimensional subspace of $T_{x} M, l \geq 2$, and $\left\{e_{1}, \cdots, e_{l}\right\}$ be an orthonormal basis of $L$. Then the Casorati curvature of the $l$-plane section $L$ with respect to the semi-symmetric metric connection is given by

$$
\mathcal{C}(L)=\frac{1}{l} \sum_{r=n+1}^{n+p} \sum_{i, j=1}^{l}\left(h_{i j}^{r}\right)^{2}
$$

The normalized $\delta$-Casorati curvatures $\delta_{c}(n-1)$ and $\hat{\delta}_{c}(n-1)$ with respect to the semi-symmetric metric connection are given by [7]

$$
\left[\delta_{\mathcal{C}}(n-1)\right]_{x}=\frac{1}{2} \mathcal{C}_{x}+\frac{n+1}{2 n} \inf \left\{\mathcal{C}(L) \mid L \text { a hyperplane of } T_{x} M\right\}
$$

and

$$
\left[\hat{\delta}_{\mathcal{C}}(n-1)\right]_{x}=2 \mathcal{C}_{x}-\frac{2 n-1}{2 n} \sup \left\{\mathcal{C}(L) \mid L \text { a hyperplane of } T_{x} M\right\}
$$




\section{Main Results}

Let $(N, g, U)(\operatorname{dim} N=m)$ be a Riemannian manifold with metric $g$ and a unit vector field $U$. The structural group of this manifold is $O(m-1) \times 1 . T_{x} N$ and $\mathfrak{X} N$ will stand for the tangent space to $N$ at a point $x$ and the algebra of smooth vector fields on $N$, respectively. The 1 -form corresponding to the unit vector $U$ is denoted by $\xi$, i.e.,

$$
\xi(X)=g(U, X), \quad X \in \mathfrak{X N}
$$

The distribution of the 1 -form $\xi$ is denoted by $\Delta$, i.e., $\Delta(x)=\left\{X \in T_{x} N \mid \xi(X)=0\right\}$.

Any section $E$ in $T_{x} N$ determines an angle $\angle(E, U)$. A Riemannian manifold $(N, g, U)$, $\operatorname{dim} N \geq 3$, is said to be of quasi-constant sectional curvature if for any arbitrary 2-plane $E$ in $T_{x} N$ with $\angle(E, U)=\varphi$, the sectional curvature of $E$ only depends on the point $x$ and the angle $\varphi$.

We will consider an $(n+p)$-dimensional Riemanniann manifold $N^{n+p}$ of quasi-constant curvature endowed with a semi-symmetric non-metric connection $\bar{\nabla}$ and the Riemannian connection $\hat{\bar{\nabla}}$.

From [24], the curvature tensor $\hat{\bar{R}}$ with respect to the Levi-Civita connection $\hat{\bar{\nabla}}$ on $N^{n+p}$ is expressed by

$$
\begin{aligned}
\hat{R}(\bar{X}, \bar{Y}, \bar{Z}, \bar{W})= & a[g(\bar{X}, \bar{Z}) g(\bar{Y}, \bar{W})-g(\bar{Y}, \bar{Z}) g(\bar{X}, \bar{W})] \\
& +b[g(\bar{X}, \bar{Z}) \xi(\bar{Y}) \xi(\bar{W})-g(\bar{X}, \bar{W}) \xi(\bar{Y}) \xi(\bar{Z}) \\
& +g(\bar{Y}, \bar{W}) \xi(\bar{X}) \xi(\bar{Z})-g(\bar{Y}, \bar{Z}) \xi(\bar{X}) \xi(\bar{W})]
\end{aligned}
$$

for any vector fields $\bar{X}, \bar{Y}, \bar{Z}, \bar{W}$ on $N^{n+p}$, where $a$ and $b$ are scalar functions on $N^{n+p}$. If $b=0$, it can be easily seen that the manifold reduces to a space of constant curvature.

We assume that an $n$-dimensional Riemannian manifold $M^{n}$ is a submanifold in $N^{n+p}$. Decomposing the vector field $U$ on $M^{n}$ uniquely into its tangent and normal components $U^{\top}$ and $U^{\perp}$, respectively, we have

$$
U=U^{\top}+U^{\perp}
$$

Let us recall the following the definition from [25].

Definition 2. [25] Let $M^{n}$ be an $n$-dimensional submanifold of an $(n+p)$-dimensional Riemannian manifold $N^{n+p} . M^{n}$ is called invariantly quasi-umbilical if there exist $p$ mutually orthogonal unit normal vectors $e_{n+1}, \cdots, e_{n+p}$ such that the shape operators with respect to all directions $e_{r}$ have an eigenvalue of multiplicity $n-1$ and that for each $e_{r}$ the distinguished eigendirection is the same.

The following is our main result.

Theorem 3. Let $M^{n}, n \geq 3$, be an n-dimensional submanifold in a Riemanniann manifold $N^{n+p}$ of quasi-constant curvature endowed with a semi-symmetric metric connection. Then we have:

(i) The normalized $\delta$-Casorati curvature $\delta_{c}(n-1)$ satisfies

$$
\rho \leq \delta_{\mathcal{C}}(n-1)+a+\frac{2 b}{n}\left\|U^{\top}\right\|^{2}-\frac{2}{n} \lambda
$$

Moreover, if $P$ is tangent to $M^{n}$, the equality case of Equation (5) holds if and only if $M^{n}$ is an invariantly quasi-umbilical submanifold in $N^{n+p}$, such that with respect to suitable orthonormal tangent frame $\left\{e_{1}, \cdots, e_{n}\right\}$ and normal orthonormal frame $\left\{e_{n+1}, \cdots, e_{n+p}\right\}$, the shape operators $A_{r}=A_{e_{r}}$, $r \in\{n+1, \cdots, n+p\}$, take the following forms: 


$$
A_{n+1}=\left(\begin{array}{cccccc}
a & 0 & 0 & \cdots & 0 & 0 \\
0 & a & 0 & \cdots & 0 & 0 \\
0 & 0 & a & \cdots & 0 & 0 \\
\vdots & \vdots & \vdots & \ddots & \vdots & \vdots \\
0 & 0 & 0 & \cdots & a & 0 \\
0 & 0 & 0 & \cdots & 0 & 2 a
\end{array}\right), A_{n+2}=\cdots=A_{n+p}=0
$$

(ii) The normalized $\delta$-Casorati curvature $\hat{\delta}_{\mathcal{c}}(n-1)$ satisfies

$$
\rho \leq \hat{\delta}_{c}(n-1)+a+\frac{2 b}{n}\left\|U^{\top}\right\|^{2}-\frac{2}{n} \lambda
$$

Moreover, if $P$ is tangent to $M^{n}$, the equality case of Equation (6) holds if and only if $M^{n}$ is an invariantly quasi-umbilical submanifold in $N^{n+p}$, such that with respect to suitable orthonormal tangent frame $\left\{e_{1}, \cdots, e_{n}\right\}$ and normal orthonormal frame $\left\{e_{n+1}, \cdots, e_{n+p}\right\}$, the shape operators $A_{r}=A_{e_{r}}$, $r \in\{n+1, \cdots, n+p\}$, take the following forms:

$$
A_{n+1}=\left(\begin{array}{cccccc}
2 a & 0 & 0 & \cdots & 0 & 0 \\
0 & 2 a & 0 & \cdots & 0 & 0 \\
0 & 0 & 2 a & \cdots & 0 & 0 \\
\vdots & \vdots & \vdots & \ddots & \vdots & \vdots \\
0 & 0 & 0 & \cdots & 2 a & 0 \\
0 & 0 & 0 & \cdots & 0 & a
\end{array}\right), A_{n+2}=\cdots=A_{n+p}=0
$$

Remark 1. For $b=0$, Theorem 3 is due to Theorem 1.1 in [7].

The proof of this theorem will be given after the following two lemmas.

Lemma 4. Let $f\left(x_{1}, x_{2}, \cdots, x_{n}\right)$ be a function in $\mathbb{R}^{n}$ defined by

$$
f\left(x_{1}, x_{2}, \cdots, x_{n}\right)=n \sum_{i=1}^{n-1} x_{i}^{2}+\frac{n-1}{2} x_{n}^{2}-2 \sum_{1 \leq i<j \leq n} x_{i} x_{j}
$$

If $x_{1}+x_{2}+\cdots+x_{n}=\varepsilon$, then we have

$$
f\left(x_{1}, x_{2}, \cdots, x_{n}\right) \geq 0,
$$

where the equality holds if and only if

$$
x_{1}=x_{2}=\cdots=x_{n-1}=\frac{1}{2} x_{n}=\frac{1}{n+1} \varepsilon
$$

Proof. A simple calculation yields

$$
\begin{aligned}
f\left(x_{1}, x_{2}, \cdots, x_{n}\right) & =n \sum_{i=1}^{n-1} x_{i}^{2}+\frac{n-1}{2} x_{n}^{2}-\left[\left(\sum_{i=1}^{n} x_{i}\right)^{2}-\left(x_{1}^{2}+x_{2}^{2}+\cdots+x_{n}^{2}\right)\right] \\
& =(n+1) \sum_{i=1}^{n-1} x_{i}^{2}+\frac{n+1}{2} x_{n}^{2}-\varepsilon^{2}
\end{aligned}
$$


On the other hand, using the Cauchy inequality we have

$$
\left(\varepsilon-x_{n}\right)^{2}=\left(\sum_{i=1}^{n-1} x_{i}\right)^{2} \leq(n-1) \sum_{i=1}^{n-1} x_{i}^{2}
$$

where the equality holds if and only if $x_{1}=x_{2}=\cdots=x_{n-1}$.

Combining Equations (7) and (8), we have

$$
\begin{aligned}
f\left(x_{1}, x_{2}, \cdots, x_{n}\right) & \geq \frac{n+1}{n-1}\left(\varepsilon-x_{n}\right)^{2}+\frac{n+1}{2} x_{n}^{2}-\varepsilon^{2} \\
& =\frac{1}{2(n-1)}\left[(n+1) x_{n}-2 \varepsilon\right]^{2} \\
& \geq 0
\end{aligned}
$$

which represents Lemma 4 to prove.

The following lemma can be proved in a similar way.

Lemma 5. Let $f\left(x_{1}, x_{2}, \cdots, x_{n}\right)$ be a function in $\mathbb{R}^{n}$ defined by

$$
f\left(x_{1}, x_{2}, \cdots, x_{n}\right)=\frac{2 n-3}{2} \sum_{i=1}^{n-1} x_{i}^{2}+2(n-1) x_{n}^{2}-2 \sum_{1 \leq i<j \leq n} x_{i} x_{j}
$$

If $x_{1}+x_{2}+\cdots+x_{n}=\varepsilon$, then we have

$$
f\left(x_{1}, x_{2}, \cdots, x_{n}\right) \geq 0
$$

where the equality holds if and only if

$$
x_{1}=x_{2}=\cdots=x_{n-1}=2 x_{n}=\frac{2}{2 n-1} \varepsilon
$$

Proof of Theorem 3 Now, we are ready to prove the theorem.

Let $x \in M^{n}$ and $\left\{e_{1}, e_{2}, \cdots, e_{n}\right\}$ and $\left\{e_{n+1}, \cdots, e_{n+p}\right\}$ be orthonormal bases of $T_{x} M^{n}$ and $T_{x}^{\perp} M$, respectively. From Equations (2)-(4) we have

$$
\begin{aligned}
R_{i j i j}= & a+b\left[g\left(U^{\top}, e_{i}\right)^{2}+g\left(U^{\top}, e_{j}\right)^{2}\right]+g\left(h\left(e_{i}, e_{i}\right), h\left(e_{j}, e_{j}\right)\right)-g\left(h\left(e_{i}, e_{j}\right), h\left(e_{i}, e_{j}\right)\right) \\
& -\alpha\left(e_{i}, e_{i}\right)-\alpha\left(e_{j}, e_{j}\right)
\end{aligned}
$$

which implies

$$
2 \tau=n^{2} H^{2}-n \mathcal{C}+\left(n^{2}-n\right) a+2 b(n-1)\left\|U^{\top}\right\|^{2}-2(n-1) \lambda
$$

Consider the following function $\mathcal{P}$ which is a quadratic polynomial in the components of the second fundamental form:

$$
\mathcal{P}=\frac{1}{2} n(n-1) \mathcal{C}+\frac{(n-1)(n+1)}{2} \mathcal{C}(L)-2 \tau+\left(n^{2}-n\right) a+2 b(n-1)\left\|U^{\top}\right\|^{2}-2(n-1) \lambda
$$

Assuming, without loss of generality, that $L$ is spanned by $e_{1}, e_{2}, \cdots, e_{n-1}$, which together with Equation (9) gives 


$$
\begin{aligned}
\mathcal{P}= & \sum_{r=n+1}^{n+p}\left\{n \sum_{i=1}^{n-1}\left(h_{i i}^{r}\right)^{2}+\frac{n-1}{2}\left(h_{n n}^{r}\right)^{2}+2(n+1) \sum_{1 \leq i<j \leq n-1}\left(h_{i j}^{r}\right)^{2}\right. \\
& \left.+(n+1) \sum_{i=1}^{n-1}\left(h_{i n}^{r}\right)^{2}-2 \sum_{1 \leq i<j \leq n} h_{i i}^{r} h_{j j}^{r}\right\} \\
\geq & \sum_{r=n+1}^{n+p}\left\{n \sum_{i=1}^{n-1}\left(h_{i i}^{r}\right)^{2}+\frac{n-1}{2}\left(h_{n n}^{r}\right)^{2}-2 \sum_{1 \leq i<j \leq n} h_{i i}^{r} h_{j j}^{r}\right\}
\end{aligned}
$$

On the other hand, we can set

$$
h_{11}^{r}+\cdots+h_{n n}^{r}=k^{r}
$$

where $k^{r}$ are real constants. In fact, for a fixed normal vector $e_{r}, k^{r}$ is the trace of the matrix $\left(h_{i j}^{r}\right)$, which is invariant no matter how the entries $h_{i j}^{r}$ change.

Then by Lemma 4 ,

$$
\mathcal{P} \geq 0
$$

with the equality case holds if and only if

$$
h_{11}^{r}=h_{22}^{r}=\cdots=h_{n-1, n-1}^{r}=\frac{1}{2} h_{n n}^{r}
$$

Combining Equations (10) and (11) and the definition of $\delta_{c}(n-1)$, we can derive inequality Equation (5). The equality case of Equation (5) holds if and only if

$$
h_{11}^{r}=h_{22}^{r}=\cdots=h_{n-1, n-1}^{r}=\frac{1}{2} h_{n n}^{r} ; h_{i j}^{r}=0, i \neq j
$$

Moreover, if $P$ given by Equation (1) is tangent to $M$, by using Lemma 1, we have

$$
\hat{h}_{11}^{r}=\hat{h}_{22}^{r}=\cdots=\hat{h}_{n-1, n-1}^{r}=\frac{1}{2} \hat{h}_{n n}^{r} ; \hat{h}_{i j}^{r}=0, i \neq j
$$

i.e., $M^{n}$ is invariantly quasi-umbilical.

Considering the following quadratic polynomial in the components of the second fundamental form

$$
\mathcal{Q}=2 n(n-1) \mathcal{C}+\frac{1}{2}(n-1)(1-2 n) \mathcal{C}(L)-2 \tau+\left(n^{2}-n\right) a+2 b(n-1)\left\|U^{\top}\right\|^{2}-2(n-1) \lambda
$$

and combining Equation (9), we have

$$
\begin{aligned}
\mathcal{Q}= & \sum_{r=n+1}^{n+p}\left\{\frac{2 n-3}{2} \sum_{i=1}^{n-1}\left(h_{i i}^{r}\right)^{2}+2(n-1)\left(h_{n n}^{r}\right)^{2}+(2 n-1) \sum_{1 \leq i<j \leq n-1}\left(h_{i j}^{r}\right)^{2}\right. \\
& \left.+2(2 n-1) \sum_{i=1}^{n-1}\left(h_{i n}^{r}\right)^{2}-2 \sum_{1 \leq i<j \leq n} h_{i i}^{r} h_{j j}^{r}\right\} \\
\geq & \sum_{r=n+1}^{n+p}\left\{\frac{2 n-3}{2} \sum_{i=1}^{n-1}\left(h_{i i}^{r}\right)^{2}+2(n-1)\left(h_{n n}^{r}\right)^{2}-2 \sum_{1 \leq i<j \leq n} h_{i i}^{r} h_{j j}^{r}\right\} \\
\geq & 0
\end{aligned}
$$

here we used Lemma 5 . Then by the very definition of $\hat{\delta}_{c}(n-1)$, we can easily derive the inequality Equation (6). Also, the equality case can be easily verified. 
Acknowledgments: The authors were supported in part by Natural Science Foundation in Anhui (No.1608085MA03) and NSF for Higher Education in Anhui (No. KJ2014A257). More over, we are thankful to the referees for their valuable comments and suggestions which improved the paper.

Author Contributions: Pan Zhang suggested to establish Casorati inequalities for submanifolds in a Riemannian manifold of quasi-constant curvature with a semi-symmetric metric connection. Liang Zhang checked the calculations and polished the draft.

Conflicts of Interest: The authors declare no conflict of interest.

\section{References}

1. Chen, B.Y. Mean curvature and shape operator of isometric immersions in real space forms. Glasg. Math. J. 1996, 38, 87-97.

2. Chen, B.Y. Geometric and topological obstructions to various immersions in submanifold theory and some related open problems. Kragujev. J. Math. 2015, 39, 93-109.

3. Casorati, F. Nuova definitione della curvatura delle superficie e suo confronto con quella di Gauss. Rend. Inst. Matem. Accad. Lomb. 1889, 22, 1867-1868. (In Italian)

4. Decu, S.; Haesen, S.; Verstraelen, L. Optimal inequalities involving Casorati curvatures. Bull. Transylv. Univ. Brasov, Ser. B 2007, 14, 85-93.

5. Decu, S.; Haesen, S.; Verstraelen, L. Optimal inequalities characterising quasi-umbilical submanifolds. J. Inequalities Pure Appl. Math. 2008, 9, 1-7.

6. Ghişoiu, V. Inequalities for the Casorati curvatures of slant submanifolds in complex space forms. In Proceedings of the RIGA 2011 Riemannian Geometry and Applications, Bucharest, Romania, 10-14 May 2011; pp. 145-150.

7. Lee, C.W.; Yoon, D.W.; Lee, J.W. Optimal inequalities for the Casorati curvatures of submanifolds of real space forms endowed with semi-symmetric metric connections. J. Inequalities Appl. 2014, 2014, 327, doi:10.1186/1029-242X-2014-327.

8. Lee, C.W.; Yoon, D.W.; Vîlcu, G.E.; Lee, J.W. Optimal inequalities for the Casorati curvatures of submanifolds of generalized space forms endowed with semi-symmetric metric connections. Bull. Korean Math. Soc. 2015, 52, 1631-1647.

9. Lee, J.W.; Vîlcu, G.E. Inequalities for generalized normalized $\delta$-Casorati curvatures of slant submanifolds in quaternionic space forms. Taiwan. J. Math. 2015, 19, 691-702.

10. Slesar, V.; Sahin, B.; Vîlcu, G.E. Inequalities for the Casorati curvatures of slant submanifolds in quaternionic space forms. J. Inequalities Appl. 2014, 2014, 123, doi:10.1186/1029-242X-2014-123.

11. Lee, C.W.; Lee, J.W.; Vîlcu, G.E. A new proof for some optimal inequalities involving generalized normalized $\delta$-Casorati curvatures. J. Inequalities Appl. 2015, 2015, 310, doi:10.1186/s13660-015-0831-0.

12. Zhang, P.; Zhang, L. Inequalities for Casorati curvatures of submanifolds in real space forms. 2014, arXiv:1408.4996 [math.DG].

13. Zhang, P.; Zhang, L. Remarks on inequalities for the Casorati curvatures of slant submanifolds in quaternionic space forms. J. Inequalities Appl. 2014, 2014, 452.

14. Oprea, T. Optimization methods on Riemannian submanifolds. An. Univ. Bucur. Mat. 2005, 54, 127-136.

15. Mihai, A.; Özgür, C. Chen inequalities for submanifolds of real space forms with a semi-symmetric metric connection. Taiwan. J. Math. 2010, 14, 1465-1477.

16. Mihai, A.; Özgür, C. Chen inequalities for submanifolds of complex space forms and Sasakian space forms endowed with semi-symmetric metric connections. J. Math. 2011, 41, 1653-1673.

17. Özgür, C.; Murathan, C. Chen inequalities for submanifolds of a locally conformal almost cosymplectic manifold with a semi-symmetric metric connection. An. St. Univ. Ovidius Constunta 2010, 18, 239-254.

18. Özgür, C.; Murathan, C. Chen inequalities for submanifolds of a cosymplectic space form with a semi-symmetric metric connection. Ann. Alexandru Ioan Cuza Univ. Math. 2012, 58, 395-408.

19. Özgür, C. BY Chen inequalities for submanifolds a Riemannian manifold of a quasi-constant curvature. Turk. J. Math. 2011, 35, 501-509.

20. Zhang, P.; Zhang, L.; Song, W. Chen's inequalities for submanifolds of a Riemannian manifold of quasi-constatnt curvature with a semi-symmetric metric connection. Taiwan. J. Math. 2014, 18, 1841-1862.

21. Yano, K. On semi-symmetric metric connection. Rev. Roum. Math. Pures Appl. 1970, 15, 1579-1586. 
22. Nakao, Z. Submanifolds of a Riemannian manifold with semi-symmetric metric connections. Proc. Am. Math. Soc. 1976, 54, 261-266.

23. Imai, T. Notes on semi-symmetric metric connections. Tensor (N.S.) 1972, 24, 293-296.

24. Chen, B.Y.; Yano, K. Hypersurfaces of a conformally flat space. Tensor (N.S.) 1972, 26, 318-322.

25. Chen, B.Y. Geometry of Submanifolds; Marcel Dekker, Inc.: New York, NY, USA, 1973.

(C) 2016 by the authors; licensee MDPI, Basel, Switzerland. This article is an open access article distributed under the terms and conditions of the Creative Commons by Attribution (CC-BY) license (http:/ / creativecommons.org/licenses/by/4.0/). 